\title{
Aspectos atuais na formação e preparação dos profissionais da saúde frente aos cuidados paliativos
}

Current aspects in the training and preparation of health professionals regarding palliative

\author{
Juliete Venancio de Sousa $^{1 \sigma}$, Gabriel Coutinho Gonçalves ${ }^{2}{ }^{\circ}$, Dennys Ramon de Melo Fernandes \\ Almeida $^{3 \sigma}$, Rafaella Bastos Leite ${ }^{3 \sigma}$, Juliana Campos Pinheiro ${ }^{3^{*}} \bullet$, Anairtes Martins de Melo ${ }^{16}$ \\ ${ }^{1}$ Centro Universitário UniFanor, Fortaleza, Ceará, Brasil. ${ }^{2}$ Centro de Ciências Sociais, Universidade Estadual do Ceará, Fortaleza, Ceará, \\ Brasil. ${ }^{3}$ Centro de Biociências, Universidade Federal do Rio Grande do Norte, Natal, Rio Grande do Norte, Brasil. *Autor para \\ correspondência. E-mail: julianapinheiroodonto92@gmail.com
}

\begin{abstract}
Resumo: Introdução: Os cuidados paliativos (CP) se comportam como uma filosofia que promove uma qualidade de vida aos pacientes em seus estágios de doença progressiva e terminal, proporcionando suporte nas dimensões física, mental e espiritual, sem dispensar a atenção familiar de cada indivíduo. O objetivo desse estudo é apresentar através de uma revisão integrativa da literatura, a atuação dos profissionais da área da saúde nos cuidados paliativos, demonstrando a importância de uma equipe multiprofissional neste âmbito. Revisão: A humanização nos cuidados paliativos consiste em atender as necessidades do paciente, sendo ele com diagnóstico de doença crônica terminal ou não, dessa forma, a humanização e os cuidados paliativos têm relação em conceito e prática, devendo ser iniciados desde a fase inicial da doença até sua fase avançada. Quando se trata da composição da equipe de profissionais, esta deve conter médicos, psicólogos, enfermeiros, fisioterapeutas, assistentes sociais e terapeuta ocupacional, pois esses profissionais assumem o cuidado especializado com foco no atendimento do paciente de forma individualizada, pois cada indivíduo reage de forma diferente a uma terapêutica, sendo as ações intervencionistas de curto ou longo prazo. Considerações finais: O processo de finitude de vida requer que os profissionais da saúde estejam preparados não só para a competência prática. Porém, existem sinais de ausência do ensino e das abordagens pedagógicas na temática de $\mathrm{CP}$, abordar essa temática durante a graduação do aluno da área da saúde, irá agregar questões fundamentais, tanto para o âmbito profissional e pessoal, tornando-o empático para lidar com pacientes que enfrentam o fim da vida, oferecendo respeito e os cuidados necessários.
\end{abstract}

Palavras-chave: cuidados paliativos, fisioterapia, docentes, ensino.

\begin{abstract}
Introduction: Palliative care (PC) behaves like a philosophy that promotes quality of life for patients in their stages of progressive and terminal illness, providing support in the physical, mental and spiritual dimensions, without giving up the individual attention of each individual. The objective of this study is to present, through an integrative literature review, the performance of health professionals in palliative care, demonstrating the importance of a multidisciplinary team in this area. Review: Humanization in palliative care consists of meeting the needs of the patient, whether he is diagnosed with a terminal chronic disease or not, thus, humanization and palliative care are related in concept and practice, and must be initiated from the initial stage of the disease to its advanced stage. When it comes to the composition of the team of professionals, it must contain doctors, psychologists, nurses, physiotherapists, social workers and occupational therapists, as these professionals assume specialized care with a focus on patient care individually, as each individual reacts differently. different to a therapeutic one, with short or long term interventional actions. Final Remarks: The finitude of life process requires health professionals to be prepared not only for practical competence. However, there are signs of absence of teaching and pedagogical approaches in the theme of PC, addressing this theme during the graduation of the student in the health area, will add fundamental issues, both for the professional and personal scope, making it empathic to deal with patients facing the end of life, offering respect and the necessary care.
\end{abstract}

Keywords: palliative care, physiotherapy, teachers, teaching.

\section{Introdução}

A Organização Mundial de Saúde (OMS), em conceito definido em 1990 e atualizado em 2002, ressalta que os cuidados paliativos consistem na promoção de uma assistência por uma equipe multidisciplinar com o 
objetivo de melhorar a qualidade de vida do paciente e de seus familiares no enfrentamento de uma doença ameaçadora da vida. Podem-se utilizar meios que previnam e aliviem o sofrimento, tratando a dor e os demais sintomas físicos, sociais, psicológicos e espirituais (INCA, 2018). Os cuidados paliativos (CP) se comportam como uma filosofia de promover a qualidade de vida aos pacientes em seus estágios de doença progressiva e terminal, proporcionando um suporte nas dimensões física, mental e espiritual, sem dispensar a atenção familiar de cada indivíduo (Gomes \& Othero, 2016). Silva (2017) relata que os cuidados paliativos não são baseados em protocolos, mas em princípios, trazendo a importância da vida ameaçada e possibilitando uma intervenção abrangente desde os aspectos físicos, psicossociais e espirituais, sem esquecer da importância do cuidado com a família no processo de morte do paciente.

O maior objetivo dos cuidados paliativos é possibilitar um melhor tempo de vivência possível aos doentes e assistir suas famílias, sendo esses cuidados introduzidos também no período de luto (Maciel et al., 2006). Portanto, este estudo objetiva-se através de uma revisão integrativa da literatura, apresentar a atuação dos profissionais da área da saúde nos cuidados paliativos, demonstrando a importância de uma equipe multiprofissional neste âmbito, e demonstrando também a importância da preparação do aluno de graduação, para lidar com pacientes que necessitem desses cuidados, oferecendo-os os melhores suportes em sua vida profissional.

\section{Revisão}

De acordo com Maciel et al. (2006), o vocábulo “paliativo” provém do latim Pallium, que retrata um manto que foi usado pelos peregrinos nos períodos de viagens a santuários para se protegerem das intempéries. Em analogia, o termo "cuidado paliativo" refere-se ao cuidar do indivíduo doente no seu período de fim de vida. Dessa forma, os cuidados paliativos precisam ser ofertados no período inicial das doenças crônicas com potencial final, para que os últimos dias de vida não se tornem árduos. Em 1960, no Reino Unido, a Médica e também assistente social e Enfermeira Cicely Saunders tornou-se a pioneira da temática em cuidados paliativos, seu movimento incluiria a assistência, o ensino e a pesquisa, tendo um marco da sua trajetória a criação Christophers Hospice em Londres de 1967, e ainda na década de 1970, a Psiquiatra Elisabeth KüblerRoss aderiu esse movimento, por meio de seus conhecimentos com Cicely, difundindo-o na América, onde foi fundado um hospice, e assim expandindo e disseminando os cuidados paliativos para diversos países (Gomes \& Othero, 2016).

Historicamente o tema Cuidados Paliativos, no Brasil, foi iniciado nos anos 70 e aparecendo com mais ênfase nos anos 90, neste de forma mais experimental, estando o Prof. Marco Túlio de Assis Figueiredo entre os pioneiros do serviço no país, juntamente com Instituto Nacional do Câncer (INCA), do Ministério da Saúde (MS). Em 1986, deu início, na unidade IV do INCA, um serviço que se dedicara exclusivamente aos cuidados paliativos (Carvalho \& Parsons, 2012). No ano de 2000 houve um crescimento significativo com a consolidação dos serviços já existentes e a fundação de outros. O Hospital do Servidor Público Estadual de São Paulo (HSPE/SP) agregou o programa de CP e dois anos após, em 2002, no mesmo hospital, surgiu a enfermaria que seria comandada pela Dra. Maria Goretti Sales Maciel, reforçando que o HSPE/SP foi também um dos agregadores para as intervenções com CP. Ressalta-se que este projeto deu início no ano de 2001 e em junho de 2004 foi realmente inaugurado e comandado pela Dra. Dalva Yukie Matsumoto (Carvalho \& Parsons, 2012). O surgimento da Academia Nacional de Cuidados Paliativos deu-se no ano de 2005, em que a partir do avanço importante na temática para o Brasil, houve a regularização do profissional paliativista, ajudando assim na elaboração de duas resoluções que regulamenta a prática médica em CP (Carvalho \& Parsons, 2012). Ainda como fato histórico no Brasil, em 2009, o Conselho de Medicina incluiu em seu código de ética médica os CP como princípio fundamental. Na atualidade, a ANCP busca a regularização da Medicina Paliativa na forma de lei e prevê um avanço nos próximos anos em relação à temática no país (Carvalho \& Parsons, 2012).

Bifulco e Iochida (2006) descreveram que a humanização nos cuidados paliativos consiste em atender as necessidades do paciente, sendo ele com diagnóstico de doença crônica terminal ou não, dessa forma, a humanização e os cuidados paliativos têm relação em conceito e prática, devendo ser iniciados desde a fase inicial da doença até sua fase avançada. Quando se trata da composição da equipe de profissionais, esta deve conter médicos, psicólogos, enfermeiros, fisioterapeutas, assistentes sociais e terapeuta ocupacional, pois esses profissionais assumem o cuidado especializado com foco no atendimento do paciente de forma individualizada, pois cada indivíduo reage de forma diferente a uma terapêutica, sendo as ações 
intervencionistas de curto ou longo prazo. O termo humanização, refere-se ao cuidar e tornar humano, favorecer a condição humana e humanizar. Percebe-se a partir disso, o desafio do cuidar paliativo no que se refere a formar uma equipe de profissionais paliativistas, pois o cuidar no formato de olhar o doente, não só pela doença ou pela finitude, gera a responsabilidade de proporcionar o alívio dos sintomas, tratar sentimentos e perturbações referentes ao findar da vida e assim gerar uma ação ao cuidado integral do ser humano (Silveira, Ciampone, \& Gutierrez, 2014). Silveira et al. (2014) abordaram que uma boa atividade de estreitamentos de relações entre paciente e profissional gera satisfação em comum, oferecendo para a vida do paciente uma melhora da qualidade de vida, e para o profissional do prazer em contribuir para o bem-estar.

Costa-Filho et al. (2008) descreveram que o Brasil dispõe de poucas unidades de assistência em CP dentro dos hospitais, sendo observado que há inúmeros problemas relacionados aos cuidados com o paciente, como a dificuldade de comunicação entre profissionais e familiares, evidenciando o despreparo por parte dos profissionais ao depararem-se com pacientes em situação de finitude. Todavia, apesar da dificuldade revelada em tal cenário, é possível perceber que ocorrem estratégias que habilitam o profissional a lidar com paciente sem prognóstico de cura. Cada vez mais, o CP domiciliar intensifica-se pela proposta de atividade humanizada com cuidados e assistência a pacientes e familiares que enfrentam a situação de terminalidade de vida. Essa modalidade de cuidado abrange atividade de monitorização do paciente, tratamento e controle de sintomatologias físicas e ainda de cuidados aos aspectos psicossociais e espirituais, acrescenta-se que o manejo dessas atividades pode ocorrer por meio de cuidadores formais, ou como também pode ser exercida por algum membro do corpo familiar (Duarte, Fernandes, \& Freitas, 2013). Ainda discorreram sobre a importância da capacitação do cuidador familiar para o direcionamento de atividades, com auxílio, orientação e apoio de profissionais no manejo de pacientes sem prognósticos de cura, buscando favorecer a continuidade de bons cuidados e a melhora da qualidade de vida do paciente e dos seus familiares (Duarte et al., 2013).

Pinheiro (2010) acredita que a implantação de uma disciplina para cuidados paliativos poderia beneficiar os estudantes de graduação em saúde na percepção dos cenários contidos na prática diária de sua atuação, por meio de um ensino focado na visão humanística. Diante de discentes que não conhecem nem mesmo a definição de cuidados paliativos determinada pela OMS, a modificação na grade curricular viabilizaria a formação de profissionais mais preparados na abordagem da terminalidade da vida. A formação em cuidados paliativos não é suficiente dentro do ensino dos cursos de graduação da área de saúde, e isso reduz bastante o acesso ao conhecimento sobre o tema, dificultando o aprimoramento da classe quando se fala dos pacientes terminais. Costa, Poles e Silva (2016) ainda ressaltam a importância da criação de disciplinas obrigatórias para os cursos, promovendo habilidades teóricas e práticas e desenvolvendo habilidades necessárias aos profissionais no enfrentamento do desafio de lidar com pacientes em estado terminal e, contudo, proporcionar uma reflexão sobre os valores da vida.

Após a graduação em Medicina, o profissional assume um papel importante na equipe de cuidados paliativos, pois ele é o responsável pelo diagnóstico e prognóstico do paciente e ainda propõe tratamentos, sendo eles medicamentosos ou não. Para tanto, além dessa tarefa, deve coordenar uma boa comunicação entre profissionais envolvidos, paciente e sua família, focalizando o bem-estar e a qualidade de vida do paciente independentemente do estágio da doença (Carvalho \& Parsons, 2012). O Código de Ética Médica (CEM) busca recuperar o assunto de formação humanística dentro das academias que aborde a formação do médico não somente com conhecimento técnico, mas também formação ética e humana para que o atendimento se torne humanizado e integral, resultando uma visão ampla de atendimento em diferentes especialidades (Chaves et al., 2011). Ainda reforçam que o médico é o profissional fundamental quando se fala em suporte para os pacientes paliativistas sem possibilidade de cura, com o intuito de promover cuidados que permitam uma qualidade de vida sem adiar e nem prolongar a morte, ressaltando a postura de assistência familiar.

Marcucci (2005) revela que a formação em graduação de Fisioterapia apresenta uma deficiência quando se aborda a temática CP no campo de trabalho, pois a formação em Fisioterapia, muitas vezes, está voltada para as estratégicas de intervenção técnica, desconsiderando a formação ética e humanista necessária aos atendimentos de cuidados paliativos, para aqueles discentes ou profissionais que tenham ou despertem um interesse maior para o aprofundamento da temática em discussão. A Fisioterapia nos cuidados paliativos iniciou suas abordagens em meados de 1960, quando o profissional passou a atuar em atendimentos a pacientes oncológicos, enfatizando que o fisioterapeuta tem uma grande contribuição nos atendimentos aos pacientes oncológicos e como um profissional com papel definido na equipe multiprofissional em CP (Girão \& Alves, 2013). 
Dentre as especialidades regulamentadas, a Fisioterapia Oncofuncional foi regulamentada a partir da Resolução 364, de 20 de maio de 2009 e modificada após dois anos pela Resolução 390/2011. Esta prevê que a Fisioterapia Oncofuncional é uma especialidade própria e exclusiva do profissional Fisioterapeuta, sendo o mesmo reconhecido com seu título de Especialista, caso cumpra os critérios estabelecidos em Resolução própria. A Resolução 397/2011 disciplina a atividade do Fisioterapeuta no exercício da Especialidade Profissional em Fisioterapia Oncológica. Essa resolução defende que o fisioterapeuta especialista profissional em Fisioterapia Oncológica, realize consulta fisioterapêutica, anamnese, interconsulta e encaminhamento, além de avaliação física e cinesiofuncional específica do paciente oncológico, solicite, aplique e interprete escalas, questionários e testes funcionais e exames complementares específicos da área oncológica, determine o diagnóstico e prognóstico fisioterapêutico, prescreva a terapêutica adequada, realize a intervenção fisioterapêutica para preservar, manter, desenvolver e restaurar a integridade cinético funcional de órgãos e sistemas em todas as fases do desenvolvimento humano, estabeleça cuidados paliativos oncológicos, prescreva, adapte e monitore as órteses, as próteses e a tecnologia assistiva, utilize os recursos de ação isolada ou concomitante de agente cinésio-mecano-terapêutico, termoterapêutico, crioterapêutico, hidroterapêutico, fototerapêutico, eletroterapêutico, sonidoterapêutico, aplique medidas de controle de infecção hospitalar, realize o posicionamento no leito, sedestação, ortostatismo, deambulação e oriente/facilite a funcionalidade do paciente oncológico, determine as condições de alta fisioterapêutica, registre em prontuário consulta, avaliação, diagnóstico, prognóstico, tratamento, evolução, interconsulta, intercorrências e alta fisioterapêutica, emita laudos, pareceres, relatórios e atestados fisioterapêuticos, planeje, participe e execute as ações vinculadas a programas nacionais para prevenção, detecção precoce, tratamento e controle do câncer e realize atividades de educação em todos os níveis de atenção à saúde, e na prevenção de riscos ambientais e ocupacionais (Girão \& Alves, 2013).

Na formação do enfermeiro preconizada pelas Diretrizes Curriculares Nacionais (DCN), mostra-se que o perfil desses profissionais deve ser de responsabilidade social, visando solucionar e reconhecer vários tipos de problemas, relacionando-os com todas esferas dimensionais do processo saúde-doença, que seja ele humano, reflexivo, crítico e que veja o ser humano de uma forma integral (Guimarães et al., 2018). Guimarães et al. (2018) ressalta ainda, que há um despreparo dos acadêmicos de enfermagem na compreensão de atender pacientes em cuidados paliativos baseada na Lei de Diretrizes Bases (LDB) da educação, no capítulo IV, artigo 43, onde se enfatiza que o entendimento dentro dos centros de ensino devem aprimorar o conhecimento sobre as problemáticas da vida cotidiana, para que esses futuros profissionais venham se especializar para atender seus possíveis pacientes com um serviço de qualidade. $O$ perfil da equipe de enfermagem tem evidência fundamental em pacientes sem possibilidades terapêuticas de cura, pois seu campo de atuação está desde um atendimento físico geral até atendimentos com relações interpessoais, tornando-se necessária a preparação desses profissionais nessas questões do processo de finitude de vida (Susaki, Silva, \& Possari, 2006).

Ainda há evidências, no estudo de Susaki et al. (2006), que a maioria dos enfermeiros pesquisados não possuíam treinamento na área de $\mathrm{CP}$, entretanto experiências foram adquiridas para compor o aprendizado na temática por meio de formações extracurriculares, ou seja, todas fora do âmbito acadêmico em graduação. Mostrando-se assim a carência da preparação e a necessidade do aprimoramento desta temática na graduação. Portanto, há necessidade de enfatizar o ensino da temática nos cursos de graduação em enfermagem para estimular a compreensão desses profissionais ao lidarem com o processo de morte. O papel do enfermeiro, no que corresponde aos cuidados paliativos, inclui estratégias de técnicas curativas de lesões cutâneas, ações de higiene e medidas de conforto do enfermo, sendo a comunicação um fator indispensável para compor uma boa atuação do enfermeiro. A comunicação envolve enfermeiro, equipe paliativista e família, o que torna esse encargo fundamental para melhor atuação na área, tendo por objetivo principal os cuidados ao paciente dentro de suas condições de doença e finitude (Carvalho \& Parsons, 2012). O grupo de enfermagem precisa compreender os estágios do processo de finitude, pois o cuidado com esses pacientes deve ser individualizado e visto em toda sua totalidade, além de tudo, o enfermeiro deve compreender as necessidades do paciente, oferecendo suporte tanto de cuidados técnicos, como com orientações, sendo esses para doentes e familiares (Silva, 2017). Entretanto, Silva (2017) ainda relatou os desafios encontrados por enfermeiros quando o assunto é paciente paliativista, destacando que muitos deles não conseguem dividir o lado profissional do lado pessoal, fazendo-se necessária, por vezes, a intervenção médica ou até mesmo o uso de medicamentos antidepressivos. 
Em março de 2000, foi fundado o Laboratório de Estudos sobre a Morte (LEM), sendo ele parte do Departamento de Psicologia da Aprendizagem, do Desenvolvimento e da Personalidade, do Instituto de Psicologia da USP. Sua criação permitiu realizar a integração no processo de formação de alunos em graduação, profissionais, docentes e pós-graduandos, que tem como um dos objetivos estimular a busca de conhecimento, reflexão e discussão sobre o tema da morte e também contribuir para a formação de profissionais de saúde e educação, tornando-os sensíveis à prestação de cuidados com pessoas que estão vivendo situações de perda, limite e morte, diante das várias fases do desenvolvimento do processo de fim de vida, assim formando pessoas capacitadas para o cuidado de forma humanística (Susaki et al., 2006). O psicólogo está inserido dentro do corpo que compete a atenção em cuidados paliativos. Existem poucos estudos relatando o papel do psicólogo na atuação paliativista, possivelmente devido a filosofia ter iniciado na Inglaterra, onde o psicólogo não atua na equipe multiprofissional. Porém, como um bom contribuinte para os cuidados paliativos, o psicólogo tem competência plena para o desenvolvimento de estratégias que evitam transtornos de desequilíbrio, sofrimento e angústia aos pacientes, devido a desconfortos ocasionados pelo processo de adoecimento (Ferreira, Lopes, \& Melo, 2011).

Batista e Gonçalves (2011) apreciam o assunto de Política de Educação Permanente em Saúde e trazem como proposta um trabalho pedagógico com uso de metodologias ativas ao ensino em saúde. Tais metodologias são baseadas na pedagogia crítico-reflexiva, envolvendo a problematização para articular diferentes realidades. Sobre o papel do aluno e a função do professor na construção do saber, é importante colocar que o processo de educação de adultos pressupõe a utilização de metodologias ativas de ensinoaprendizagem, que proponham desafios a serem superados pelos participantes, que lhes possibilitem ocupar o lugar de sujeitos na construção dos conhecimentos e que coloquem o professor como facilitador e orientador desse processo (Batista, \& Gonçalves, 2011). Em 1990, na Escola de Direito de Harvard, houve a primeira abordagem pedagógica em que foi empregado o método de caso, em que os convidados participavam de apresentações de casos e problemáticas de suas organizações e, posteriormente, houve uma expansão das estratégias de ensinos para outras instituições (Fávero, Villar, \& Walter, 2016). Os mesmos autores descrevem que existem várias formas de abordagens de ensino, dentre elas, as técnicas de leitura, que visam as discussões em salas de aula, seminários e ainda artigos, cujos conteúdos são apropriados pelo discente, ampliando conhecimentos específicos e de caráter científico, tornando-o preparado para o mercado de trabalho. Diesel, Baldez e Martins (2017) perceberam em seu estudo que os discentes ao receberem abordagens de ensino tradicional, isto é, discente apenas como um receptor, relatam desinteresses em aulas rotineiras e comuns, por serem enfadonhas e não estimulantes. Com base nesse aspecto, viram ser viável que os docentes desenvolvam estratégias metodológicas que atraiam seu público, pois as várias formas de ministrar o conteúdo geram interesse no discente para participar das abordagens de ensino.

\section{Discussão}

Skaba (2005) aborda as contribuições acerca do ato de humanização em relação aos cuidados paliativos, no sentido de manter uma relação interdisciplinar de aproximação entre profissional e paciente, enfatizando a importância do profissional rever suas atitudes diante das ações com indivíduos em sua finitude, e ainda destaca que a base dos cuidados paliativos deve ser focada na humanização. Os cuidados paliativos domiciliares intensificam-se pela proposta de atividade humanizada com cuidados e assistência a pacientes e familiares que enfrentam a situação de terminalidade de vida. Essa modalidade de cuidado abrange atividade de monitorização do paciente, tratamento e controle de sintomatologias físicas e ainda de cuidados aos aspectos psicossociais e espirituais, acrescenta-se que o manejo dessas atividades pode ocorrer por meio de cuidadores formais, ou como também pode ser exercida por algum membro do corpo familiar (Duarte et al., 2013). Ainda discorreram sobre a importância da capacitação do cuidador familiar para o direcionamento de atividades, com auxílio, orientação e apoio de profissionais no manejo de pacientes sem prognósticos de cura, buscando favorecer a continuidade de bons cuidados e a melhora da qualidade de vida do paciente e dos seus familiares (Duarte et al., 2013).

Para Hermes e Lamarca (2013), o profissional médico assume o compromisso de orientar os demais integrantes da equipe no que diz respeito ao paciente terminal, e isso exige conhecimento além do tecnicismo percorrendo o autoconhecimento, necessitando por muitas vezes de apoio físico e psicológico. A fisioterapia nos cuidados paliativos contribui de forma bastante abrangente, pois é uma profissão que tem um campo 
amplo de estratégias quando o assunto são os cuidados para com os usuários. Suas técnicas não se restringem à recuperação, pois também contemplam o tratamento personalizado, que vai desde a promoção à prevenção da enfermidade (Girão \& Alves, 2013). A estratégica fisioterapêutica desenvolve-se por meio de uma diversidade de procedimentos que vai desde uma boa avaliação à reavaliação, em que se objetiva cuidar do utente em vários aspectos, e promover o bem-estar físico e mental está entre essas condutas (Girão\& Alves, 2013). Dentre inúmeras condutas fisioterapêuticas que podem ser utilizadas, estão terapias que visam trazer uma melhor qualidade de vida. Dentre as condições vividas pelo usuário, há recursos para alívio de dor, como as correntes elétricas que beneficiará com efeitos de analgesia, que em muitos casos diminuem até o uso de medicamentos para minimizar as dores articulares e musculares. Técnicas que promovem relaxamento muscular são de extrema importância, pois aliviam os sintomas psicofísicos e estão entre as modalidades usadas para ter respostas positivas para esses pacientes, tal como atuação nas complicações osteomioarticulares, pulmonares e linfáticas (Marcucci, 2005).

A atuação da enfermagem com pacientes paliativistas consiste no conhecimento do processo da fisiopatologia da doença, no entendimento de fármacos e da fisiologia humana como meio de condutas de controle dos sintomas do paciente. Além disso, foi visto que os enfermeiros sentem necessidade de aprendizado, e postulam a inclusão dessas temáticas nos cursos de graduação, pois admitem sentirem-se despreparados para lidar com essas circunstâncias (Hermes \& Lamarca, 2013). A compreensão sobre cuidados paliativos parte do princípio de análise da visão do terapeuta ao observar o paciente em sua totalidade, notando-se a necessidade de um maior aprimoramento sobre a temática desses cuidados. As experiências ocorrem e são compreendidas desde o período de formação, o que torna de suma importância o desenvolvimento do processo de confronto com a terminalidade de vida durante o período de graduação, podendo assim evitar possíveis desprazeres durante a vida profissional desses estudantes (Scharamm, 2002).

Sabe-se do grande desafio em abordar diferentes metodologias dentro da sala de aula, tendo sempre em mente a responsabilidade de integrar o conhecimento teórico-prático. Partindo desse suposto, alguns estudos enfocam o uso de metodologias com jogos, na atribuição do ensino da temática de $\mathrm{CP}$, pelo fato de que os estudantes podem vivenciar situações e enfrentar circunstâncias fora do tradicional âmbito de graduação, adquirindo influências, exposições e experiências sucessivas com a morte e o luto no contexto clínico da temática de CP (Alonso et al., 2018).

\section{Considerações finais}

O processo de finitude de vida requer que os profissionais da saúde estejam preparados não só para a competência prática. Porém, existem sinais de ausência do ensino e das abordagens pedagógicas na temática de $\mathrm{CP}$, abordar essa temática durante a graduação do aluno da área da saúde, irá agregar questões fundamentais, tanto para o âmbito profissional e pessoal, tornando-o empático para lidar com pacientes que enfrentam o fim da vida, oferecendo respeito e os cuidados necessários.

\section{Referências}

Alonso, A. I. L., Martínez, M. E. F., Presa, C. L., Casares, A. M. V., \& González, M. P. C. 2018. Los juegos experimentales de aula: una herramienta didáctica en cuidados paliativos. Revista da Escola de Enfermagem da USP, 52(1), e03310.

Andrade, C. G., de Melo Alves, A. M. P., da Costa, S. F. G., \& Santos, F. S. 2014. Cuidados paliativos ao paciente em fase terminal. Revista Baiana de Enfermagem, 28(2), 126-133.

Aristizábal, J. F., Martínez-Smit, R., Díaz, C., \& Pereira Filho, V. A. 2018. Surgery-first approach with 3D customized passive self-ligating brackets and 3D surgical planning: Case report. Dental Press Journal of Orthodontics, 23(3), 47-57.

Batista, K. B. C., \& Gonçalves, O. S. J. 2011. Formação dos profissionais de saúde para o SUS: significado e cuidado. Saúde e Sociedade, 20, 884-899.

Bifulco, V. A., \& Iochida, L. C. 2009. A formação na graduação dos profissionais de saúde e a educação para o cuidado de pacientes fora de recursos terapêuticos de cura. Revista Brasileira de Educação Médica, 33(1), 92-100. 
Carvalho, R. T., \& Parsons, H. A. 2012. Manual de Cuidados Paliativos ANCP. Academia Nacional de Cuidados Paliativos, 2(1), 23-25.

Chaves, J. H. B., Mendonça, V. L. G. D., Pessini, L., Rego, G., \& Nunes, R. 2011. Cuidados paliativos na prática médica: contexto bioético. Revista Dor, 12(3), 250-255.

Costa Filho, R. C., Costa, J. L. F., Gutierrez, F. L. B. D. R., \& Mesquita, A. F. D. 2008. Como implementar cuidados paliativos de qualidade na unidade de terapia intensiva. Revista Brasileira de Terapia Intensiva, 20(1), 88-92.

Costa, Á. P., Poles, K., \& Silva, A. E. 2016. Formação em cuidados paliativos: experiência de alunos de medicina e enfermagem. Interface-Comunicação, Saúde, Educação, 20(59), 1041-1052.

Diesel, A., Baldez, A. L. S., \& Martins, S. N. 2017. Os princípios das metodologias ativas de ensino: uma abordagem teórica. Revista Thema, 14(1), 268-288.

Duarte, I. V., Fernandes, K. F., \& Freitas, S. C. D. 2013. Cuidados paliativos domiciliares: considerações sobre o papel do cuidador familiar. Revista da SBPH, 16(2), 73-88.

Fávero, J. D., Villar, E. G., \& Walter, S. A. 2016. Desenvolvimento do ensino de estratégia em cursos de graduação em Administração de Instituições de Ensino Superior pela perspectiva de professores e alunos. Revista Gestão Universitária na América Latina-GUAL, 9(2), 70-94.

Ferreira, A. P. D. Q., Lopes, L. Q. F., \& Melo, M. C. B. D. 2011. O papel do psicólogo na equipe de cuidados paliativos junto ao paciente com câncer. Revista da SBPH, 14(2), 85-98.

Girão, M., \& Alves, S. 2013. Fisioterapia nos cuidados paliativos. Revista de Ciências da Saúde da ESSCVP, 5, 34-41.

Gomes, A. L. Z., \& Othero, M. B. 2016. Cuidados paliativos. Estudos avançados, 30(88), 155-166.

Guimarães, C. 2018. Três décadas de educação profissional em Saúde. Um balanço das prioridades e dos investimentos na formação de técnicos para o SUS. Revista Poli - Saúde, Educação e Trabalho, 59(1), 20-21.

Hermes, H. R., \& Lamarca, I. C. A. 2013. Cuidados paliativos: uma abordagem a partir das categorias profissionais de saúde. Ciência \& Saúde Coletiva, 18, 2577-2588.

Instituto Nacional de Câncer [INCA]. 2018. Cuidados Paliativos. Rio de Janeiro, RJ: INCA.

Maciel, M. G. S., Rodrigues, L. F., Naylor, C., Bettega, R., Barbosa, S. M., Burlá, C., \& Melo, I. T. V. 2006. Critérios de qualidade para os cuidados paliativos no Brasil. Academia Nacional de Cuidados Paliativos, 1(1), 60.

Marcucci, F. C. I. 2005. O papel da fisioterapia nos cuidados paliativos a pacientes com câncer. Revista Brasileira de Cancerologia, 51(1), 67-77.

Pinheiro, T. R. S. P. 2010. Avaliação do grau de conhecimento sobre cuidados paliativos e dor dos estudantes de medicina do quinto e sexto anos. O Mundo da Saúde, 34(3), 320-6.

Scharamm, F. R. 2002. Morte e finitude em nossa sociedade: implicações no ensino dos cuidados paliativos. Revista Brasileira de Cancerologia, 48(1), 17-20.

Silva, L. M. O. 2017. Atuação dos enfermeiros frente aos cuidados paliativos a pacientes oncológicos pediátricos. Monografia (Bacharel em enfermagem). Fortaleza, CE: Faculdades Nordeste.

Silveira, M. H., Ciampone, M. H. T., \& Gutierrez, B. A. O. 2014. Percepção da equipe multiprofissional sobre cuidados paliativos. Revista Brasileira de Geriatria e Gerontologia, 17(1), 7-16.

Skaba, M. F. 2005. Humanização e cuidados paliativos. Ciência \& Saúde Coletiva, 10(3), 782-784.

Susaki, T. T., Silva, M. J. P. D., \& Possari, J. F. 2006. Identificação das fases do processo de morrer pelos profissionais de Enfermagem. Acta Paulista de Enfermagem, 19(2), 144-149.

\section{Minicurrículo}

Juliete Venancio de Sousa. Possui graduação em FISIOTERAPIA pelo Centro Universitário Faculdades Nordeste - UNIFANOR (2018). Tem experiência na área de Fisioterapia, atuando principalmente nos seguintes temas: Terapia Aquática, Reabilitação, Pós-Operatório, Cuidados Paliativos e Saúde Coletiva. 
Gabriel Coutinho Gonçalves. Fisioterapeuta. Especialista em Residência Multiprofissional em Saúde da Família. Especialista em Micropolitica na Gestão e no Trabalho em Saúde. Mestrando em Planjamento e Políticas Públicas (UECE). Centro de Estudos Sociais Aplicados - Programa de Políticas Públicas.

Dennys Ramon de Melo Fernandes Almeida. Possui especialização em Micropoliticas da Gestão e do Trabalho em Saúde pela Universidade Federal Fluminense (UFF). É mestre em Patologia pela Universidade Federal do Ceará (UFC) e doutorando em Ciências Odontológicas pela Universidade Federal do Rio Grande do Norte (UFRN). Tem experiência na área de Odontologia atuando principalmente nos seguintes temas: atenção primária em saúde, ensino superior, lesões periapicais e neoplasias de cavidade oral. Atualmente é membro da Sociedade Brasileira de Estomatologia e Patologia Oral.

Rafaella Bastos Leite. Cirurgiã-dentista, graduada pela Universidade Estadual da Paraíba. Mestre em Patologia Oral pela Universidade Estadual da Paraíba; Doutorado em Ciências Odontológicas- área de concentração: Patologia Oral- UFRN.

Juliana Campos Pinheiro. Cirurgiã-Dentista graduada pela Universidade Tiradentes. Mestre em Patologia Oral pela Universidade Federal do Rio Grande do Norte (UFRN); Doutorado em Ciências Odontológicas (andamento)- área de concentração: Biologia Experimental- UFRN (2018-2022); Membro da Sociedade Brasileira de Estomatologia e Patologia Oral (2016-atualmente).

Anairtes Martins de Melo. Possui graduação em fisioterapia pela Universidade de Fortaleza (UNIFOR) e mestrado em Ensino na Saúde pela Universidade Estadual do Ceará (UECE). Atualmente é professora mestre do Centro Universitário Fanor/ Unifanor Wyden. Tem experiência na área de Educação, com ênfase em educação de nível superior, atuando principalmente nos seguintes temas: Fisioterapia, Idosos, Tecnologia em saúde, Gerontologia e sub área: acidentes por queda.

Como citar: Sousa, J.V., Gonçalves, G.C., Almeida, D.R.M.F., Leite, F.B., Pinheiro, J.C., \& Melo, A.M. 2020. Aspectos atuais na formação e preparação dos profissionais da saúde frente aos cuidados paliativos. Pubsaúde, 3, a045. DOI: https://dx.doi.org/10.31533/pubsaude3.a045

Recebido: 17 jun. 2020.

Revisado e aceito: 14 jul. 2020.

Conflito de interesse: os autores declaram, em relação aos produtos e companhias descritos nesse artigo, não ter interesses associativos, comerciais, de propriedade ou financeiros que representem conflito de interesse.

Licenciamento: Este artigo é publicado na modalidade Acesso Aberto sob a licença Creative Commons Atribuição 4.0 (CC-BY 4.0). 